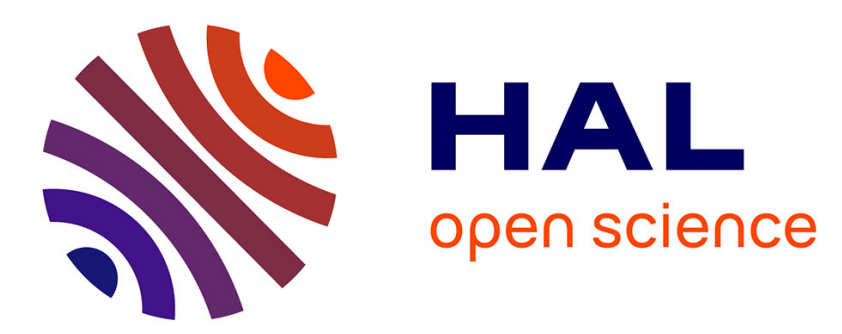

\title{
Faire face aux comportements perturbants: le travail de contrainte en milieu hospitalier gériatrique
}

\author{
Lucie Lechevalier Hurard
}

\section{To cite this version:}

Lucie Lechevalier Hurard. Faire face aux comportements perturbants : le travail de contrainte en milieu hospitalier gériatrique. Sociologie du Travail, 2013, 55 (3), pp.279-301. 10.1016/j.soctra.2013.07.006 . hal-00860872

\section{HAL Id: hal-00860872 \\ https://hal.science/hal-00860872}

Submitted on 11 Sep 2013

HAL is a multi-disciplinary open access archive for the deposit and dissemination of scientific research documents, whether they are published or not. The documents may come from teaching and research institutions in France or abroad, or from public or private research centers.
L'archive ouverte pluridisciplinaire HAL, est destinée au dépôt et à la diffusion de documents scientifiques de niveau recherche, publiés ou non, émanant des établissements d'enseignement et de recherche français ou étrangers, des laboratoires publics ou privés. 


\title{
Faire face aux comportements perturbants :
}

\section{le travail de contrainte en milieu hospitalier gériatrique}

\author{
Lucie Lechevalier Hurard1,2
}

\section{Résumé :}

Au delà des formulations médiatiques et professionnelles qui ont retenu le lexique de la «maltraitance » et de la «bientraitance » pour qualifier et traiter les phénomènes de violence dans la prise en charge des personnes âgées vulnérables, l'article vise à restituer un pan des pratiques des soignants en milieu hospitalier gériatrique que cette terminologie aux accents prescriptifs laisse dans l'ombre. Le travail de contrainte apparaît comme partie intégrante de l'activité quotidienne de l'équipe de l'unité de soins de longue durée qui a fait l'objet de l'enquête ethnographique mobilisée ici.

Le cas particulier des patients âgés dont les comportements perturbent l'ordre hospitalier est examiné car il confronte les soignants à des cas limites où la mobilisation de la contrainte ne va pas de soi.

L'objectif est de faire apparaître les processus de négociation au cours desquels les professionnels élaborent des normes communes qui tracent des frontières entre des gestes de contrainte maîtrisés et assumés collectivement d'un côté et des dérapages inconsidérés et condamnables de l'autre.

Mots clé : contrainte, violence, maltraitance/bientraitance, démence, maladie d'Alzheimer, hôpital, prise en charge des personnes âgées, négociation, légitimation.

Avis : ce texte est la version de l'auteur d'un article qui a été accepté pour publication dans la revue Sociologie du Travail. Les modifications résultant du processus de publication (et notamment la mise en page) ne sont pas reprises dans le présent document.

\footnotetext{
${ }^{1}$ Doctorante Université Paris 13/IRIS, ATER Université de Bretagne Occidentale, lucielh@yahoo.com

2 Je souhaite remercier vivement les membres du jury du Prix du jeune auteur 2012 ainsi que les personnes qui, par leurs discussions ou leurs relectures, m'ont accompagnée dans l'élaboration de la réflexion présentée dans ce texte : les participants au séminaire des doctorants de l'Iris, Christelle Avril, Delphine Serre, Delphine Moreau, Violaine Roussel, Yasmine Bouagga, Benjamin Derbez, Aude Béliard, Iris Loffeier et Benoît Eyraud.
} 
La «maltraitance » s'est imposée, dans les écrits des professionnels (Gérontologie et société, 2010 ; Hugonot, 2007) mais aussi des chercheurs en sciences sociales (Dujarier, 2002 ; Molinier, 2009), comme terminologie privilégiée pour aborder le problème de la violence dans le champ de la prise en charge des personnes âgées et dans celui des milieux de soins, notamment hospitaliers. Cette dénomination correspond moins à une spécification de la nature de certains actes de brutalité, qui serait propre aux milieux du soin aux personnes vulnérables, qu'à la description d'une configuration relationnelle dans laquelle s'inscrivent ces actes. Ce terme souligne, sur le registre de la condamnation morale, l'illégitimité et l'intolérable du recours à la violence, dans un contexte où celui qui l'exerce détient un mandat de protection sur celui qui la subit, rendu vulnérable et sans défense par sa maladie, son âge ou sa dépendance physique et psychique.

Concernant le contenu des actes désignés, la notion de maltraitance à l'encontre de personnes vulnérables a un caractère «malléable et expansionniste » (Hacking, 1988, p.54 ; Vigarello, 2005). Elle ne désigne aujourd'hui plus les seuls phénomènes de violence physique mais englobe une faisceau élargi d'actes voire d'omissions ou de négligences qui ont, selon la définition retenue par le Conseil de l'Europe (Brown, 2002) et reprise par une Commission d'enquête du Sénat Français (Juilhard et Blanc, 2003), "pour effet de porter gravement atteinte, que ce soit de manière volontaire ou involontaire, aux droits fondamentaux, aux libertés civiles, à l'intégrité corporelle, à la dignité ou au bien-être général d'une personne vulnérable » (Brown, 2002, p.9).

La qualification de "maltraitance ordinaire», adoptée par les auteures d'un rapport rédigé pour la Haute Autorité de Santé, marque le succès d'un lexique qui s'applique désormais à des actes «banals», "invisibles», faisant l'objet d'une «acceptation passive » (Compagnon et Ghadi, 2009, p.1), incluant jusqu'à de «petites négligences quotidiennes : faire des toilettes trop rapides, ne pas répondre aux appels des personnes, servir des repas trop tôt, ne pas respecter les rythmes de chacun pour le lever et le coucher... $»^{3}$.

L'augmentation de la sensibilité générale au phénomène et la diffusion dans le grand public du terme vont de pair. Les affaires de maltraitance en institutions

3. Intervention de Philippe Bas, ministre délégué à la Sécurité sociale, aux Personnes âgées, aux Personnes handicapées et à la famille, conférence de presse Plan bientraitance/maltraitance, 14 mars 2007. 
pour personnes âgées sont fortement médiatisées et les familles n'hésitent plus à manifester leur condamnation d'actes qui jusqu'alors passaient inaperçus. Parallèlement à cette expansion se développe une approche préventive qui prend, de manière antonymique, le nom de «politiques de la bientraitance » (HAS 2012). L'ANESM $^{4}$, dans l'une de ses premières recommandations, annonce sa volonté d'expliciter en priorité la notion de bientraitance avant de travailler à des recommandations spécifiques sur la maltraitance, afin d'«aborder les pratiques professionnelles sous un angle positif» (ANESM, 2008, p.1). Pourtant, la formulation du débat dans les termes de cette dichotomie est perçue par certains soignants comme " un étau stigmatisant » (Deliot et al, 2009, p.597) et une mise en accusation de leurs pratiques du soin. La «culture de la bientraitance» (ANESM, 2008, p.1) est dénoncée comme un «système décontextualisé de normes et de principes abstraits à valeur générale», (Deliot et al., 2009, p.595). Produite par des bureaucrates éloignés des conditions réelles du soin, elle serait relayée par des gestionnaires d'établissements qui méconnaissent le caractère lui-même nuisible de l'institution qu'ils dirigent et en particulier de l'organisation du travail en son sein. Dans les discussions des soignants à l'hôpital, le stigmate est retourné et les maltraitants deviennent des maltraités, cette dynamique en cascade étant invoquée pour expliquer l'irruption de gestes brutaux vis-à-vis des personnes âgées hospitalisées.

Le rapport de la Haute Autorité de Santé s'inscrit dans cette perspective causale : les actes de maltraitance y sont envisagés comme le fait d'un individu seul qui « dérape » « à un moment donné » (Compagnon et Ghadi, 2010, p.69). Individuels le plus souvent, ils apparaissent donc comme ponctuels et accidentels. Des dysfonctionnements organisationnels, liés notamment au manque récurrent de personnel qualifié, seraient à la source de ces comportements déviants par rapport à la norme professionnelle établie de bientraitance. Dans le rapport, les membres de l'encadrement interrogés (directeurs d'hôpitaux et cadres de santé) se décrivent comme les garants de la surveillance des gestes des soignants qui sont sous leur

\footnotetext{
${ }^{4}$ L'ANESM (Agence nationale de l'évaluation et de la qualité des établissements et services sociaux et médico-sociaux) est un Groupement d'intérêt public qui a été créée en 2007 pour accompagner la mise en œuvre de l'évaluation interne et externe instituée par la loi du 2 janvier 2002 sur la rénovation de l'action sociale et médico-sociale. Elle produit depuis 2008 des recommandations de bonnes pratiques professionnelles sur différents sujets et a fait de sa recommandation sur la bientraitance le cadre de référence de l'ensemble de ses publications.
} 
responsabilité afin qu'ils appliquent au mieux cette nouvelle norme hospitalière.

A partir d'une approche des situations de travail et de l'activité d'une équipe de soignants dans une unité hospitalière gériatrique de soins de longue durée (USLD), je propose ici d'entrer dans les pratiques concrètes des professionnels de la prise en charge en institution de personnes âgées considérées comme démentes ou atteintes de la maladie d'Alzheimer.

\section{Présentation de l'enquête ethnographique et des données mobilisées}

L'enquête ethnographique mobilisée ici a été menée entre juillet 2009 et mars 2010 dans l'une des unités de soins de longue durée (USLD) d'un hôpital gériatrique public de région parisienne, accueillant 80 patients pour des durées allant de quelques semaines à plusieurs années.

Les salariés dont le travail est décrit ici sont majoritairement des « soignants ». Cette catégorie générique désigne en général dans les services hospitaliers l'ensemble des intervenants qui pratiquent des soins, allant des actes infirmiers ou de nursing aux diverses activités thérapeutiques voire occupationnelles. Pour rompre avec le caractère euphémique et homogénéisant du terme de "soignants", qui a tendance à gommer les rapports hiérarchiques existant entre les différents statuts professionnels qui constituent la catégorie, je distinguerai le groupe des « soignants de première ligne » (aides-soignants, infirmiers) des autres (psychologue, psychomotricienne, animatrice, cadre de santé etc.).

Parmi ces soignants, plusieurs statuts d'emploi coexistent dans le service enquêté. Les salariés de l'équipe exerçant sur les mêmes postes (en particulier aides-soignants et infirmiers) peuvent être indifféremment, sans que le contenu attendu de leur travail en soit affecté, agents titulaires de la fonction publique hospitalière, agents stagiaires (ayant obtenu le concours et dans l'attente d'une titularisation à l'issue d'une année de stage), contractuels en CDI ou CDD de plus ou moins longue durée (certains travaillent dans le service depuis plusieurs années, d'autres sont embauchés seulement pour les remplacements de congés annuels) ou intérimaires recrutés à la journée (par le 
biais d'agences d'intérim spécialisées).

Les soignants de première ligne sont le plus souvent titulaires d'un diplôme d'Etat (aide-soignant ou infirmier) ou équivalent. Cela n'est cependant pas le cas des élèves des écoles du secteur sanitaire (dans le cadre de leurs stages professionnels ils pratiquent les soins mais n'assument pas la charge complète d'un poste de travail) ni des contractuels remplaçant les aides-soignants, recrutés au moment des congés annuels (dans l'USLD enquêtée, ils ne disposent d'aucune formation dans le secteur sanitaire).

Plusieurs membres de l'équipe soignante, titulaires de diplômes étrangers du secteur sanitaire non reconnus en France, exercent dans l'équipe à un grade inférieur: les médecins comme infirmiers, les infirmiers comme aidessoignants. Les médecins du service sont eux aussi titulaires de diplômes étrangers et perçoivent des salaires inférieurs à ceux des médecins hospitaliers français durant une période d'exercice de trois ans, nécessaire à la validation de leur diplôme. En dehors de ces cas particuliers, les salaires sont encadrés par la grille indiciaire de la fonction publique hospitalière, avec une majoration liée à une organisation en roulements qui implique pour les soignants de première ligne de travailler en moyenne deux dimanches par mois.

Les données ont été recueillies principalement par observation participante des activités quotidiennes des soignants (présents au moins à moyen terme dans l'équipe, les élèves, intérimaires et contractuels de courte durée n'ayant pas été suivis de manière intensive) ainsi que des réunions de l'équipe pluridisciplinaire (qui rassemblent aides-soignants, infirmiers, médecins, cadres de santé, assistante sociale, psychomotricienne, psychologue, animatrice, représentant de l'accueil de jour et diététicienne). L'observation a été complétée par une dizaine d'entretiens formels et informels menés avec les personnels soignants, médicaux et paramédicaux et par l'accès aux dossiers d'hospitalisation et dossiers médicaux de l'ensemble des patients ainsi qu'au logiciel de transmission.

Les données ethnographiques présentées ont été anonymisées. Elles sont suivies d'une référence entre crochets indiquant soit la date à laquelle elles figurent dans le journal de terrain (JT) soit la date de l'entretien (ent.). 
Le cadre de compréhension en termes de bientraitance et de maltraitance, qui structure les débats professionnels et politiques, sera laissé de côté au profit d'une analyse de ce que j'appellerai le travail de contrainte qui se réalise dans le service. Il ne s'agit pas ici d'opérer une simple substitution lexicale. Les actes qui peuvent être qualifiés de maltraitance et ceux qui composent le travail de contrainte constituent d'ailleurs des ensembles distincts, même s'ils se recoupent partiellement. Ainsi, des menaces verbales ou l'utilisation massive de psychotropes peuvent se retrouver dans les deux catégories. Cependant, les négligences, l'inattention aux besoins fondamentaux de la personne, les coups physiques ou les abus sexuels n'entrent pas sous la catégorie de travail de contrainte. Cette dernière, plus restreinte, inclut aussi des formes de rappel à l'ordre ou de confinement qui ne sont généralement pas qualifiées de maltraitance par les professionnels du secteur. On entend ainsi par travail de contrainte un ensemble de pratiques, mises en œuvre par l'équipe hospitalière et encadrées par des négociations formelles et informelles entre les professionnels, qui visent à maîtriser les corps des patients dans la perspective de leur administrer des soins.

Parler ici de contrainte comme mode de traitement officiel - alors que le monde hospitalier distingue la maltraitance, dérapage condamnable, de la contention ${ }^{5}$ permet de souligner la continuité qui existe entre des pratiques habituellement segmentées par les implicites normatifs d'un débat professionnel placé dans la sphère morale. À travers une description des interactions les plus ordinaires, on découvrira comment la contrainte, qui prend des formes très variées, constitue une part non négligeable de l'activité quotidienne des soignants de l'unité, pris entre un fonctionnement hospitalier rigide et des comportements inadéquats de patients âgés dont les corps débordent inexorablement du cadre de l'institution.

Le terme de travail prend quant à lui le contrepied de l'argument accidentel et met à l'inverse en lumière le caractère récurrent et organisé (voire obligatoire) de la contrainte. En parlant de «travail sentimental» (Strauss et al., 1982) ou « émotionnel » (Hochschild, 1979; Mercadier, 2002; Druhle, 2000), de

5. La «contention physique passive» (CPP) désigne «l'utilisation de tous les moyens, méthodes, matériels ou vêtements, qui empêchent ou limitent les capacités de mouvement volontaire de tout ou d'une seule partie du corps, dans le seul but d'obtenir la sécurité pour une personne qui présente un comportement estimé dangereux ou mal adapté»(ANAES, 2000, p.10). Elle se distingue de la contention posturale et de la contention active, à visée rééducative, qui sont en général réalisées par un kinésithérapeute. 
«travail de care » (Molinier, 2005) ou de « compétences relationnelles » (Arborio, 2000, p.135), d'autres auteurs ont décrit des formes discrètes de l'activité des salariés de l'hôpital (Molinier, 2006), du soin aux personnes ou plus largement des emplois de services. Ces savoir-faire, développés et mobilisés dans des contextes professionnels, ont en commun le peu de formalisation et de reconnaissance dont ils font l'objet, mais surtout leur caractère indispensable dans la chaîne de réalisation des tâches pour que l'institution mène ses missions à bien.

Si le travail de contrainte est lui aussi discret, en ce qu'il est peu visible pour un œil extérieur, il a pourtant cette particularité d'être plus réglementé et contrôlé que le travail sentimental ou relationnel par exemple. Sa réalisation, qui prend place dans une division hiérarchisée des tâches, n'est pas consensuelle et elle va parfois à l'encontre des valeurs personnelles des soignants. Dans certaines de ses formes, ce travail de contrainte suppose en effet une implication physique et morale de soignants, qui l'estiment pénible et pas toujours légitime, quand son utilisation ne s'inscrit pas à première vue dans une perspective d'efficacité thérapeutique.

Dans un contexte où l'accusation extensive de violence ou de maltraitance plane sur l'activité des soignants comme un soupçon qu'il s'agit de conjurer (Moreau, 2011), je m'intéresserai aux processus de négociation au cours desquels l'équipe élabore, malgré les divergences de points de vue et d'implication dans ce travail, des formes de contrainte légitimes, acceptables collectivement et mises en œuvre par tous les soignants. On verra comment se formulent, dans le quotidien de la prise en charge hospitalière de patients âgés considérés comme déments, des normes professionnelles qui tracent des frontières entre des gestes maîtrisés et assumés collectivement d'un côté et des dérapages inconsidérés et condamnables de l'autre.

\section{Travailler à la disponibilité de corps débordants}

Dans la répartition de l'offre d'hébergement et de soins aux personnes âgées, les unités hospitalières de soins de longue durée (USLD) représentent l'échelon le plus médicalisé de la prise en charge disponible : elles s'adressent en théorie à des patients dits "polypathologiques », qui nécessitent un suivi médical constant. Le public accueilli dans l'USLD étudiée présente cependant pour l'essentiel un besoin 
d'aide plutôt lié une altération des facultés mentales qu'à des incapacités physiques: la majorité des dossiers médicaux fait état de symptômes de « démence » ou de «maladie d'Alzheimer», qui, couplés ou non à des affections somatiques, rendent difficile le maintien à domicile de la personne, en raison notamment des possibilités réduites d'accompagnement dont elle dispose.

\subsection{Travailler sur les corps}

Le caractère hospitalier de la prise en charge ${ }^{6}$, couplé à la présence prédominante de patients considérés comme déments ou atteints de la maladie d'Alzheimer contribue à ce que les soins prodigués dans le service se focalisent sur une attention aux besoins du corps.

Contrairement aux maisons de retraite, qui affichent la vocation domestique de l'hébergement qu'elles offrent ${ }^{7}$ (Mallon, 2004), l'USLD étudiée, malgré des démarches pour proposer aux patients les plus valides des activités occupationnelles, apparaît essentiellement comme un lieu proposant des soins. Ce mandat est pourtant ambigu puisqu'il s'agit d'un espace thérapeutique où, paradoxalement, la réhabilitation ne semble pas un horizon réaliste. Alors qu'ils sont tournés dans leur activité ordinaire vers la production de réponses médicales et techniques à l'apparition progressive de facteurs de perte d'autonomie, les soignants évoquent pour l'USLD une mission d'accompagnement sur le long terme d'un processus inéluctable de dégradation de l'état physique et mental dont l'issue est le plus souvent le décès de la personne ${ }^{8}$.

Hélène Rollin, cadre de santé, explique en réunion : « Nous on fait la fin de vie de personnes qu'on connaît depuis longtemps, qui ont une démence, qui se dégradent. » [JT 13/10/09]

6. Son architecture (des couloirs bordés par des chambres doubles, parfois individuelles, et une salle à manger pour 12 à 15 patients), la spécialisation de son personnel (le personnel médical et paramédical en USLD représente $61 \%$ des effectifs, contre $30 \%$ en moyenne dans les maisons de retraite privées par exemple, DRESS, 2005, p.8, tableau 7), l'organisation du travail par «roulement » de trois équipes et le taux d'encadrement en personnel sont caractéristiques d'un établissement hospitalier, auquel l'USLD appartient.

7. Elles s'engagent à établir les conditions nécessaires pour que le résident recrée un «chez soi » (Mallon, 2004) et pour qu'il dispose d'une certaine autonomie dans l'occupation de sa journée, tout en se voyant proposer des activités collectives qui permettent l'élargissement de sa sociabilité.

8. $69 \%$ des sorties de résidents d'USLD n'appartenant pas à des EHPAD correspondent à des décès (DRESS, 2009, p.5, tableau 6) 
Elle se distingue néanmoins d'une mission palliative intensive réalisée par des unités de soins palliatifs qu'une infirmière appelle «terminaux » [JT 13/09/09] par sa longue durée (plusieurs mois, voire plusieurs années) et par des moyens humains mis à disposition nettement moins importants.

La perception de cet accompagnement d'un déclin irrémédiable est encore accentuée lorsqu'apparaissent des symptômes d'altération cognitive dès l'hospitalisation ou au cours du séjour dans le service. Il est souvent question de la manière dont un patient a « dégringolé des paliers » ou « a descendu les marches deux par deux ». Si cette dégradation est regrettée, surtout quand elle se précipite, chacun regarde cette évolution comme ordinaire. Seuls le rythme et les formes qu'elle va prendre restent extrêmement incertains pour les soignants. D'un patient à un autre, mais aussi d'un moment à l'autre de la journée pour un même patient, les soignants du service constatent des troubles ou des symptômes variables avec lesquels ils doivent composer, pour remplir leur mission d'accompagnement.

Or, en l'absence de solutions thérapeutiques efficaces ${ }^{9}$, les interventions auprès des patients se concentrent essentiellement sur l'entretien du corps. Gérard Rimbert, dans l'enquête qu'il a menée en maison de retraite, qualifie d'ailleurs cette « exigence de soin minimale en gériatrie» de "gardiennage des corps». Elle consiste selon lui à «nourrir/vider/nettoyer les corps et à veiller à leur repos » (Rimbert, 2008, p.529) et s'apparente « à une simple surveillance des personnes et à la préservation des fonctions vitales » (Rimbert, 2010, p.187). L'expression de «gardiennage des corps», bien qu'elle rappelle la notion foucaldienne de "gouvernement des corps", est retenue par G. Rimbert pour renvoyer à la description que fait E. Goffman des institutions totales et de leur vocation de « mise en dépôt de leurs pensionnaires, comme [dans] de simples magasins » (Goffman, 2005 [1968], p.121). Effectivement, la journée du service est rythmée par la

9. Annette Leibing (2009) décrit par exemple le climat d'incertitude quant aux effets des médicaments dans lequel les médecins brésiliens prescrivent des traitements dans la prise en charge de la maladie d'Alzheimer. Elle évoque notamment les débats qui existent sur l'efficacité de ces molécules ainsi que l'évolution récente des types de traitements proposés. Alors qu'ils ciblaient dans un premier temps uniquement les atteintes cognitives, qui étaient conçues comme le cœur de la maladie, les laboratoires pharmaceutiques mettent désormais en avant des traitements des symptômes comportementaux et psychologiques qui accompagnent souvent la maladie d'Alzheimer, dans la perspective d'augmenter « la qualité de vie » et « la fonctionnalité » des patients. 
distribution en séries ${ }^{10}$ de soins, infirmiers ou d'hygiène et de confort : toilettes, habillage/déshabillage, repas, distribution de médicaments, changes, s'enchaînent et viennent rompre à intervalles réguliers, et pour quelques instants seulement, l'étirement de l'attente qui caractérise la journée d'une majorité de patients ${ }^{11}$. Ces soins sont des moments de confrontation entre «le chronomètre » et « le carillon » (Rimbert, 2005) : le premier est le temps des soignants, marqué par l'urgence, la précipitation permanente de ceux qui doivent assurer un nombre important de tâches dans un temps restreint et ne peuvent transférer à l'équipe suivante celles qui n'auraient pas été achevées ; le second est le temps domestique des patients, marqué par une certaine atonie, alors que leur seul horizon est celui de voir passer les heures de la journée.

La confrontation de ces deux temps dans les interactions quotidiennes rend évidente l'exigence première qui découle d'une organisation hospitalière rationalisée du travail: celle de la disponibilité totale des patients dans les moments où leurs corps sont sollicités pour des soins. Or, ce rapport d'utilité, dans lequel la discipline ${ }^{12}$ hospitalière inscrit les corps des malades qu'elle prend en charge, est remis en cause par les comportements de certains patients, qui apparaissent alors pour l'équipe comme perturbants.

10. L'organisation du travail hospitalier prévoit la réalisation des soins « en série » : au cours de la journée, les soins ne sont pas regroupés mais ils sont distribués successivement à chacun des patients.

11. En dehors des soins corporels, de rares activités sont proposées aux patients. Il existe un service d'animation au sein de l'hôpital et une animatrice accompagne quelques patients de l'USLD à des goûters, spectacles etc. On remarque néanmoins dans la pratique que ces activités sont essentiellement proposées aux patients qui sont suffisamment valides pour se déplacer sans assistance (et qui peuvent même aider l'animatrice pour pousser les fauteuils d'autres patients), et à ceux dont le comportement ne "pose pas problème». Dans la pratique, ce sont donc systématiquement les mêmes patients qui sortent du service pour ces activités. Il existe par ailleurs un service d'animation thérapeutique qui reçoit des patients atteints de la maladie d'Alzheimer deux journées par semaine. L'USLD concernée par l'enquête est particulièrement attentive à envoyer le plus possible de patients dans cet accueil de jour. Cependant, ces activités, là encore, ne concernent pas la majorité des patients.

12. Au sens de M. Foucault (1993 [1975], p.161) selon lequel la discipline est un ensemble de "méthodes, qui permettent le contrôle minutieux des opérations du corps, qui assurent l'assujettissement constant de ses forces et leur impose un rapport de docilité-utilité ». On peut nuancer cette définition dans le cas de la discipline en milieu hospitalier gériatrique, qui ne vise pas à majorer les aptitudes du corps dans un but productif, mais qui cherche néanmoins à limiter la lourdeur que ces corps peuvent représenter en termes d'organisation du travail de soin. . 


\subsection{Des corps débordants : comportements perturbants et rigidité hospitalière}

En général présentés comme découlant d'une atteinte cognitive pathologique (désignée sous les termes de "maladie d'Alzheimer et troubles apparentés », de "démence», de "syndrome démentiel » ou de «dégradation cognitive»), les déambulations et les fugues, comme l'agitation, l'opposition, les cris et la désinhibition sexuelle font partie des attitudes qui sont reportées dans les dossiers d'hospitalisation au titre des «troubles du comportement» considérés comme perturbants.

En effet, outre le fait qu'ils représentent un changement important de la personne par rapport à ses manières d'être antérieures, ils se révèlent inadéquats au fonctionnement hospitalier.

Le mandat des soignants de première ligne de l'USLD peut être ramené à trois types de tâches: ils doivent s'assurer du maintien des patients dans l'espace du service, garantir qu'ils reçoivent les soins prévus chaque jour, à horaire fixe et dans un temps limité, et sont enfin garants de la sérénité de la vie collective et font par conséquent en sorte que les patients ne se dérangent pas mutuellement.

C'est pourquoi, lorsque certains patients se montrent récalcitrants aux soins («opposants", dans le vocabulaire hospitalier) et qu'ils empêchent la réalisation d'un certain nombre de tâches ordinaires, ils augmentent l'incertitude pour les soignants de savoir s'ils vont pouvoir ou non effectuer les soins prescrits dans les temps impartis. Or, ceci est incompatible avec l'organisation des soins en série. D'autre part, ces comportements peuvent induire pour le personnel soignant une charge de travail supplémentaire, qui vient s'ajouter à un programme de tâches bien rempli.

Dans le service étudié, les déambulations de Mme Pougny à travers les couloirs obligent une aide-soignante à aller la chercher plusieurs fois dans la matinée, laissant en suspens la tâche à laquelle elle était occupée.

Un aide-soignant doit nettoyer les vêtements souillés de Mme Belley puis procéder à un nouveau change car elle a détaché sa protection et l'a déposée sur la table à manger.

Une infirmière se voit, elle, contrainte de repasser à plusieurs reprises pour faire son injection d'insuline à Mme Rosier car celle-ci s'y oppose fermement. 
Bien que certains soignants estiment que la gestion de ces perturbations fait partie de l'ordinaire du travail (Ines Dahmani, infirmière, explique à propos de Mme Pougny préférer «la voir comme une furie marcher dans les couloirs que dans son lit ! » [JT 29/08/09]), tout cela contribue à une augmentation de la charge de travail, dont l'organisation des soins s'accommode mal.

Dans ces cas de figure, la «simple surveillance» glisse vers une activité d'une nature différente. C'est pour décrire les interventions par lesquelles l'équipe soignante tente au mieux de conformer les corps des patients aux exigences du fonctionnement de l'institution que je parle ici de «travail de contrainte ».

\subsection{Contraintes interstitielles et contraintes prescrites}

Loin d'être forcément perçues comme violentes, illégitimes ou simplement pénibles, certaines formes que prend le travail de contrainte sont routinières.

Par exemple, la question de la circulation des patients dans l'hôpital et de sa restriction est récurrente dans le service. Bien que l'espace ne soit pas matériellement segmenté (sous la forme de portes fermées par des codes), cette circulation est limitée et les soignants de l'ensemble de l'équipe assurent collectivement un cadrage verbal pour que les patients qui déambulent soient régulièrement renvoyés « dans leur salle ${ }^{13}$, c'est à dire dans l'aile du bâtiment où se trouve leur chambre. Les déambulations tolérées sont ainsi cantonnées pour chaque patient à un couloir qui longe une douzaine de chambres et qui débouche sur une salle à manger commune.

M. Nanteuil est connu dans le service pour ses « fugues » : il est régulièrement retrouvé à l'accueil de l'établissement sur le point de sortir et, au cours de l'enquête, il a déjà disparu plusieurs jours de l'hôpital.

Au mois d'août, alors qu'elle observe une augmentation de ses tentatives de sortie, l'équipe se mobilise pour assurer un rappel verbal à l'ordre constant. Dès qu'il passe les portes (jamais fermées) des autres salles du service, le

13. Ce terme de «salle» est une réminiscence de l'ancienne structuration de l'hôpital en salles communes, qui accueillaient jusqu'au début des années 198042 puis 28 patients. Les salles communes ont été redécoupées en chambres doubles ou individuelles, les espaces communs étant désormais réduits à une salle à manger par aile de bâtiment. Ce terme de «salle » est utilisé par tous les soignants, y compris par ceux, la majorité d'entre eux, qui n'ont pas connu professionnellement cette époque de l'hôpital. 
soignant présent à ce moment-là lui demande «M. Nanteuil, qu'est-ce que vous faites?» «M. Nanteuil, où vous allez?» et lui intime l'ordre de « retourner dans sa salle » [JT 10/08/09].

Dominique Lalande, cadre de santé, se plaint d'ailleurs de devoir pallier par des moyens humains imparfaits l'absence d'un dispositif matériel qui assurerait le confinement : «C'est le problème, on est obligé de faire ça, de le rappeler tout le temps parce que c'est pas fermé ». [JT 10/08/09]

Les failles éventuelles de cette surveillance étroite opérée par les soignants sont compensées par des artifices qui font appel à une attention élargie à des tiers : pendant cette période de «fugues » régulières, les aides-soignantes habillent M. Nanteuil, habituellement en tenue de ville, avec une blouse qui indique au premier regard sa qualité de patient de l'hôpital, afin qu'il soit immédiatement repéré et ramené s'il sort de l'enceinte de l'établissement. [JT 07/08/09]

Comme dans des services d'hospitalisation psychiatrique (Moreau, 2010, p. 171), l'équipe lui retire donc, pour une période limitée dans le temps, la liberté de se vêtir avec ses effets personnels, comme il le fait ordinairement. La blouse qu'il porte ne limite pas matériellement ses déplacements mais elle le place sous une surveillance accrue de la part du personnel hospitalier mais aussi à l'extérieur de l'hôpital.

Le confinement est ici rendu effectif au moyen de rappels verbaux soutenus, qui sont perçus par les soignants comme relevant moins de la contrainte que d'une simple attention rendue particulièrement nécessaire par les comportements des patients dits « désorientés » ou « fugueurs ».

La contrainte s'exerce aussi dans le contexte de la distribution des médicaments, trois fois par jour, ainsi que lors des injections d'insuline aux patients diabétiques. Ces moments donnent en effet très régulièrement lieu à des échanges conflictuels, qui débouchent parfois sur des actes de contrainte plus soutenue, physique ou verbale.

L'une des infirmières n'hésite pas à avoir recours à des menaces explicites pour obtenir de certains patients qu'ils absorbent les médicaments qu'elle leur tend, ou à la force physique pour garantir l'immobilité de ceux auxquels elle doit faire des injections.

Quand Sonia Ichouza, infirmière, s'approche de Mme Hyppolite avec son matériel d'injection, qu'elle pose sur la table de nuit, elle lui explique très fermement qu'elle 
va devoir lui faire une piqûre. Mme Hyppolite se laisse prélever une goutte de sang sur le doigt, sans protester. Mais une fois que l'infirmière s'approche d'elle avec l'aiguille et qu'elle la met encore une fois en garde, Mme Hyppolite s'énerve. Elle jette le matériel de soins qui est sur sa table de nuit, elle gesticule. L'infirmière la réprimande d'un ton brutal: "Attention hein, si vous bougez, je demande du renfort pour vous attacher au lit, vous bougez plus, vous sortez plus, vous faites pipi caca sur vous! Attention! » [JT 28/07/09].

Mme Rosier est récemment entrée dans l'USLD. Depuis son arrivée, elle est réputée « opposante », voire violente puisque certains soignants affirment avoir reçu des coups de sa part. Pour se prémunir du danger que représente son agitation au moment d'une injection d'insuline, Sonia Ichouza sollicite l'aide d'une élève infirmière pour la maintenir immobile en l'entourant fermement de ses bras. [JT 28/07/09]

L'élève infirmière, le lendemain et les jours suivants, aura systématiquement recours à ce genre de contention ponctuelle pour réaliser l'injection, en prévention d'une éventuelle agitation.

Dans ces extraits, on voit émerger des formes de contrainte, comme le recours ponctuel à la force physique, qui engagent plus les soignants que les simples rappels verbaux. $\mathrm{Ni}$ autorisées ni prescrites, elles font partie d'une économie de petits arrangements dont la nécessité et la légitimité est négociée pour chaque cas entre les soignants qui doivent assurer cette discipline en parallèle des soins. On pourrait qualifier ces formes de contraintes d'interstitielles, étant donné leur caractère peu réglementé et la discrétion avec laquelle elles sont mises en œuvre, généralement en dehors du regard de l'extérieur (des familles de patients), voire des collègues puisque pour l'essentiel elles s'effectuent dans des circonstances où le soignant est seul avec le patient. Ces derniers ont d'ailleurs tendance à les présenter comme des manières d'accommoder leurs gestes du quotidien à des situations dont ils estiment qu'elles ne relèvent pas de leur mission de soin.

Il sera question plus loin des enjeux de légitimation qu'on distingue dans la définition d'une frontière nette entre ces gestes interstitiels et des interventions médicalement ordonnées, comme la prescription de contentions dites «mécaniques » - ceintures pelviennes ou barrières au lit qui empêchent la personne de se lever seule - ou de 
médicaments psychotropes - antidépresseurs, anxiolytiques, neuroleptiques. Mais c'est ici la continuité entre des formes de contrainte qui sont habituellement pensées séparément qui sera mise en avant, du point de vue de l'objectif qui leur est assigné.

En effet, si l'acte de prescription par un médecin renvoie a priori les contentions mécaniques et les médicaments dans l'ordre du bienfait thérapeutique, la manière dont l'équipe du service y recourt peut s'apparenter à un usage disciplinaire. Une ceinture pelvienne (dispositif en tissu qui maintient la personne assise dans son fauteuil) présente l'avantage pour les soignants de première ligne de limiter l'imprévisibilité des moments de soins. La personne retenue peut éventuellement circuler en faisant rouler son fauteuil, mais le champ de ses mouvements est borné à un périmètre restreint. Cela permet aux soignants de la trouver facilement dans les moments où ils ont besoin de lui prodiguer les soins et de la déplacer rapidement, plutôt que d'avoir à la convaincre, par exemple, de venir s'installer à table et de l'accompagner dans un transfert parfois très long. Les médicaments psychotropes ont, eux, pour effet d'atténuer l'expression des « troubles du comportement » gênants.

Ainsi, dans ses «dimensions officielles» (Avril et al., 2010, p.26-27), comme lorsqu'il prend la forme de "pratiques informelles », qu'il implique des interventions verbales ou physiques, le travail de contrainte vise toujours la même fin : obtenir la disponibilité des corps des patients nécessaire pour les soins, dans les temporalités qui sont celles de l'organisation hospitalière du travail.

Le travail de contrainte ne forme pourtant pas un tout homogène et chacun des soignants n'a pas recours à ses différentes formes à la même fréquence et avec la même intensité. Parce que sa mise en œuvre suppose une implication physique et morale forte, elle fait l'objet de négociations au cours desquelles se définit ce qui, dans des situations de prise en charge hospitalière et lorsque les patients sont considérés comme déments et atteints de troubles du comportement, est collectivement tolérable et ce qui ne l'est pas. 


\section{Légitimer le travail de contrainte}

Au cours des réunions d'équipe, mais aussi à l'occasion de discussions plus informelles, s'engagent des négociations sur les cas de différents patients dont les comportements posent problème dans le service. Dans quelles situations est-il justifié d'avoir recours à la contrainte ? Sous quelle forme ? Quels sont les mots, les gestes qui dépassent la nécessité ? Et surtout, qui doit assumer les actes de contrainte ? Qui prend la responsabilité physique, morale, mais aussi juridique, d'un travail dont beaucoup de soignants suggèrent qu'il leur répugne?

2.1 «Mais c'est une camisole !» : la contrainte dans la division organisationnelle et morale du travail

Comme celui de gardiennage des corps (Rimbert, 2010, p.187), qui a de sale le caractère trivial du traitement corporel, la proximité avec les fluides, les déjections (les aidessoignants se qualifient parfois eux-mêmes de «torche-culs »), les formes interstitielles du travail de contrainte relèvent de ce que Hughes appelle le « sale boulot» (Hughes, 1996, p.81), en raison de l'engagement physique mais aussi de la charge morale et émotionnelle qu'elles supposent.

Physiquement, la manipulation dans des temps limités de patients dont les corps sont alternativement fragiles, affaiblis, éventuellement inertes, ou à l'inverse vigoureux, récalcitrants, parfois violents, alors qu'ils ne se plient qu'assez difficilement au rythme des soins, requiert un savoir-faire corporel, des «techniques» de son propre corps (Mauss, 1968 [1934]) et de celui des patients. Certaines situations impliquent pour les soignants de mettre en jeu leur corps, de le soumettre éventuellement au danger de prendre des coups, de l'impliquer dans des gestes destinés à se protéger en même temps qu'à réaliser la tâche prévue.

Depuis mon arrivée dans le service, Diane Senely, l'une des aides-soignantes, est particulièrement loquace à mon endroit à propos de son activité. Elle est toujours très soucieuse de souligner les aspects valorisants de son travail et de la manière dont elle l'exerce.

Alors que je l'accompagne pour une matinée, elle est très avenante avec moi, 
s'avérant enthousiaste à l'idée de m'initier aux enseignements d'une formation Humanitude ${ }^{14}$ qu'elle a reçue quelques temps plus tôt. Elle m'interroge : « Ça fait partie de ta formation les toilettes? Parce que je peux te montrer comment on fait pour doucher quelqu'un, une personne démente, comment on fait pour la ramener dans notre monde, avec la méthode Humanitude ».

Elle confie à son collègue les toilettes au lit et s'engage dans une série de quatre douches réalisées avant le repas de midi. La deuxième patiente est Mme Rosier. Une fois que Diane Senely l'a amenée en fauteuil roulant jusqu'à la salle de bain, Mme Rosier refuse de se laisser faire. Elle hurle continuellement durant la vingtaine de minutes que dure la totalité de la toilette, lance ses pieds et ses mains violemment en direction de l'aide-soignante pour la frapper. Malgré cela, cette dernière réalise tous les actes d'hygiène qu'elle avait prévu de lui administrer : douche complète, lavage des cheveux, nettoyage de l'intérieur de la bouche avec du bain de bouche antiseptique, brossage et séchage des cheveux, habillage. Ces actes supposent une grande proximité physique avec Mme Rosier. Diane Senely met en œuvre un certain nombre de techniques qui lui permettent de réaliser tous ces gestes, tout en esquivant les coups de celle-ci qui lui sont destinés : elle l'arrose avec la douche à distance, s'écarte de la trajectoire des pieds qui la visent, maintient fermement les bras de Mme Rosier au niveau de ses cuisses, me demande mon assistance pour l'empêcher de se débattre pendant qu'elle lui lave l'intérieur de la bouche etc.

Après cette douche, qui a été éprouvante pour toutes les trois, Diane Senely ne m'adresse plus la parole : elle se montre très occupée, court d'un côté et de l'autre du service et n'a plus le temps pour me laisser l'accompagner dans son travail. [JT 03/09/09]

On voit apparaître ici, outre l'implication physique de cette aide-soignante, la dimension morale du «sale boulot » qu'elle effectue. Pour les soignants, il s'agit parfois de réaliser des gestes « infra-dignitate » (Hughes, 1996, p.81) qui leur répugnent, des gestes qui les

14. L'Humanitude® (Gineste et Pelissier, 2007) est une marque déposée par Yves Gineste et Rosette Marescotti qui décrit un ensemble de pratiques de soins adaptées à la prise en charge de personnes atteintes de la maladie d'Alzheimer. Les «techniques» et les « règles de l'Art » qui y sont associées sont diffusées par des formations proposées aux professionnels du secteur gérontologique, travaillant en établissement ou à domicile. Dans l'USLD étudiée, trois aides-soignantes et une cadre de santé ont suivi plusieurs journées de cette formation. 
amènent à apparaître pour eux-mêmes « dans un rôle dont ils pensent qu'ils devraient avoir un peu honte » (Hughes, 1996, p.81), du double point de vue de leurs valeurs personnelles et des normes professionnelles ${ }^{15}$ qui ont cours dans le service. Deux semaines avant cet épisode, cette même aide-soignante m'avait exprimé le sentiment de culpabilité qu'elle pouvait éprouver, dans des situations où elle avait l'impression de n'avoir pas appliqué les règles transmises lors de la formation qu'elle a reçue.

Assise devant l'ordinateur du poste de soins sur lequel elle inscrit l'ensemble des gestes qu'elle a réalisés dans la matinée, Diane Senely m'explique : «Si tu veux aller jusqu'au bout de ta formation, tu devrais faire la formation Humanitude. Tous les aides-soignants devraient passer par là. Ça te permet de gagner du temps. Parce que tu passes plus de temps avec le patient, mais ça t'en fait gagner. Tu es moins dans le stress, le speed. Si tu arrives pas à faire quelque chose, même si tu as commencé, c'est pas grave, tu reviens plus tard, ou tu expliques. Ça révolutionne la manière de travailler. Ça te fait gagner du temps dans ton travail d'abord, mais aussi personnellement. Parce que quand tu as fait les choses, que tu es seule dans ta salle, que tu as pas le temps d'appliquer la méthode, une fois que tu es devant l'ordi, c'est la culpabilité qui vient “j'aurais pas dû faire comme ça”. L’Humanitude, ça te permet de pas te sentir coupable, de savoir que tu as bien fait les choses. Mais quand on est sur une salle seul, on peut pas, on n'a pas le temps. La direction, ils le savent, ils le disent pas mais ils savent qu'ils nous maltraitent. » [JT 20/08/09]

C'est là surtout avec les normes professionnelles qui ont cours dans l'hôpital que cette aide-soignante se sent en porte-à-faux. Dans un contexte où sont désormais affichées sur les murs de tous les établissements la Charte de la personne hospitalisée (DGS, 2006) ou la Charte des droits et libertés de la personne âgée en situation de handicap ou de dépendance (FNG, 2007), les normes de bientraitance et de préservation de l'autonomie du patient apparaissent largement mises à mal par l'exercice de la contrainte, notamment lorsqu'il s'agit de s'imposer physiquement à un patient qui refuse les gestes prodigués. Ces gestes rendent évident pour les soignants le glissement qui s'est opéré

15. Didier Fassin (2009, p.1257) résume ainsi la distinction entre valeurs et normes: «les premières [renvoient] à l'appréciation de ce qui est bien et de ce qui est mal (ou meilleur et pire), les secondes se [référent] à des règles, des principes, des obligations (ce qu'il faut ou convient de faire ou ne pas faire). En réalité, si analytiquement les énoncés évaluatifs et les énoncés prescriptifs se distinguent, empiriquement la distinction est bien plus difficile à établir et probablement non pertinente car les valeurs procèdent au moins pour partie de normes et les normes dépendent pour partie de valeurs ». 
dans la forme de travail institutionnel (Laforgue, 2009) ${ }^{16}$ qu'ils sont chargés de mettre en œuvre. Alors que le milieu gériatrique valorise de plus en plus des formes d'intervention des professionnels (soignants ou non) basées sur le «travail pour autrui ${ }^{17}$, et même idéalement sur le «travail avec autrui ${ }^{18}$, l'acte de contrainte se révèle comme symptôme de la réalité des soins tels qu'ils se pratiquent en USLD et qui s'apparentent plutôt à du « travail sans autrui ». Impuissants à obtenir une réhabilitation ou une amélioration de l'état de la personne dont ils ont la charge, et alors qu'ils «ne peuvent pas non plus définir une ligne d'action à partir du point de vue des usagers » (Laforgue, 2009, §29), qui sont considérés comme déments donc incapables, certains soignants jettent l'éponge et acceptent que leur unique action possible sur ces patients soit centrée sur la logique de fonctionnement propre de l'institution. Le seul but de la prise en charge hospitalière peut alors devenir l'obtention de la résignation du patient à l'ordre institutionnel, à ses normes et à son fonctionnement, sans prendre en compte ses aspirations et même si cela doit passer par des formes d'imposition physique.

D. Laforgue insiste sur les agencements et les hybridations des différentes formes du travail institutionnel qu'on retrouve au sein de chaque institution. Dans l'hôpital observé, le travail avec/pour autrui est externalisé et réalisé hors du lieu de vie des patients, par exemple dans le service d'animation thérapeutique, dont les visées et les contraintes (en personnel notamment) sont très différentes. C'est en revanche aux soignants de première ligne de l'USLD que revient la part de «sale boulot » qu'est le «travail sans autrui ».

Cette résignation à laisser de côté la vocation de soin de l'hôpital et à avoir recours à des contraintes interstitielles est tout particulièrement décriée par les soignants

16. Denis Laforgue (2009) distingue quatre formes du travail institutionnel, avant d'analyser la manière dont elles s'agencent dans le quotidien de différentes institutions contemporaines : «travail sur autrui », « travail avec autrui », « travail pour autrui » et « travail sans autrui »

17. L'une des formulations privilégiées de ce «travail pour autrui » est le care (Paperman et Laugier, 2006), dans lequel la relation de soin traditionnellement asymétrique est rééquilibrée par une prise en compte de la vulnérabilité ontologique partagée par l' «aidant » et l' « aidé » ainsi que du point de vue de l'usager pour définir les termes de la situation et par conséquent de l'action institutionnelle (Laforgue, 2009)

18. J'utilise ici le terme dans un sens un peu différent de celui de Laforgue. Pour lui, le «travail avec autrui » revient à ce que l'institution, considérant qu'« il ne s'agit plus de normaliser mais d'accompagner des personnes en difficulté » peut limiter son action à la fixation "d'un cadre légal et réglementaire à l'intérieur duquel les acteurs concernés sont libres d'agir » §23. J'évoque ici plutôt une expression récurrente des aides-soignants qui décrivent la teneur idéale de leur intervention auprès de personnes âgées dépendantes dans les termes de l'accompagnement. La prescription est de n'intervenir que sous forme d'aide en considérant que la personne, même si elle est lente ou a des difficultés, doit être encouragée le plus possible à réaliser les gestes du quotidien elle-même, l'agent devant respecter dans une certaine mesure ses souhaits et ne faisant qu'intervenir marginalement pour pallier les incapacités qui apparaissent dans le cours de l'action. 
paramédicaux, qui sont présents dans le service seulement partiellement puisqu'ils travaillent dans différentes unités de l'hôpital.

Lors d'une réunion, l'équipe aborde le cas de Mme Belley, qui retire sans cesse ses vêtements et sa couche. Hélène Rollin, cadre de santé, suggère de proposer à la fille de la patiente de l'habiller avec une tenue qui s'attache par derrière et qu'elle ne pourra pas enlever seule. Christine Fredout, psychomotricienne, à la fois indignée de la proposition et gênée d'exprimer trop spontanément son point de vue, réagit très vivement, en utilisant le terme de «camisole » pour parler de ce vêtement, appuyée dans ce choix lexical par Aurélie Coulange, la psychologue. Elle se reprend au cours de la discussion, pour marquer sa bonne volonté à comprendre ce qui amène les aides-soignants à soutenir cette option : «Je vais changer de terme, je vais dire "l'habit", sinon c'est vraiment trop culpabilisant [...] [aux aides-soignants] j'ai besoin de votre vécu parce que moi j'ai des positions sur la camisole, mais vous qu'est-ce que vous en pensez ? » [JT 09/03/10]

On voit là comment les raisons qui motivent le recours aux contraintes interstitielles sont inégalement réparties dans l'équipe. Chacun des soignants, suivant sa position dans l'organisation du travail, le contenu des tâches qui lui sont confiées et les conditions de réalisation de celles-ci, n'en expérimente pas la même nécessité. L'usage de la contrainte interstitielle dans l'USLD est donc inscrit dans une division du travail non seulement physique mais aussi morale. En pratique, il est assigné à ceux qui sont en bas de l'échelle, c'est à dire aux aides-soignants et infirmiers, présents tout au long de la journée dans l'espace de vie des patients. Les personnels médicaux, paramédicaux et les cadres de santé interviennent ponctuellement dans la contrainte, mais généralement dans des formes qui impliquent plus la parole que le geste, plus le geste d'accompagnement ferme que celui d'imposition.

Si le travail de contrainte apparaît comme illégitime, son utilité n'est néanmoins pas contestée. C'est cette articulation problématique entre nécessité pratique et légitimité thérapeutique que l'équipe cherche à établir par des négociations, en réunion ou dans le cours des interactions quotidiennes. 
2.2 "Vous êtes là pour appliquer des prescriptions médicales»: la prescription médicale entre légitimation d'une nécessité pratique et affirmation d'une fonction thérapeutique de la contrainte

Marie-Anne Dujarier estime que dans la relation de service, dont relève le travail gériatrique, «le travail de corps à corps échappe en partie à la prescription, en même temps qu'il est [à son] fondement» (Dujarier et Gaulejac, 2006, p.27). Dans le cas du travail de contrainte, les limites et les règles de ce corps à corps ne sont codifiées et explicites qu'à partir du moment où la contention, comme acte médical, est ordonnée par un médecin. C'est cette seule prescription qui, inscrite dans le dossier du patient avec des indications quant à sa durée (forcément limitée même si des ordonnances successives peuvent rendre la mesure permanente), fait entrer le geste de contrainte dans le droit. Cette possibilité d'officialisation à travers la prescription médicale contribue à faire exister en creux la contrainte comme travail, c'est à dire comme activité nécessaire, organisée et légitime au regard du mandat confié à l'institution hospitalière et à ses salariés.

Dans l'USLD, les soignants de première ligne sont prompts à suggérer l'intervention du médecin sous forme de prescriptions de contentions mécaniques ou de médicaments.

Plusieurs aides-soignantes et infirmières discutent entre elles de la dégradation continue qu'elles constatent dans le comportement de Mme Zermati. Elles soulignent les difficultés qu'elles rencontrent : en effet, alors qu'elle a fait plusieurs chutes, qui lui ont laissé des marques importantes d'hématomes sur le visage, elles se voient obligées de la surveiller beaucoup plus qu'habituellement. Par ailleurs, les gestes de soins du quotidien se transforment désormais régulièrement en confrontations éprouvantes.

Lors de transmissions orales d'une équipe à une autre (en dehors de la présence des médecins et cadres de santé), elles s'interrogent :

Diane Senely, aide-soignante : j'espère que ça ira mieux cet après-midi, elle nous a insultés de tous les noms ce matin!

Ines Dahmani, infirmière : il faut qu'elle soit à l'unité de géronto-psychiatrie cette dame...

Diane Senely, aide-soignante : c'est nous qui allons finir à l'unité de gérontopsychiatrie si ça continue! 
Sonia Ichouza, infirmière : j'ai dit au médecin de lui mettre quelque chose [un médicament] pour la casser mais il veut pas. Elle était bien avec le Risperdal [neuroleptique]!

Diane Senely, aide-soignante : c'est une furie !

Ines Dahmani, infirmière : il faut lui mettre un balai derrière son fauteuil, la coincer dans son fauteuil en lui mettant la table devant. Loxapac, Haldol [neuroleptiques], ça ça marche ! En psychiatrie, ils mettent 50 gouttes, en gériatrie il suffit de 5 gouttes.

Karina Hernan, aide-soignante : je crois qu'il faut qu'on ait quelque chose en cas d'urgence, là où j'étais, pour les personnes Alzheimer on leur donnait une injection de quelque chose.

Ines Dahmani, infirmière : mais c'est pas une solution, il faut qu'elle voie le psychiatre et que quelque chose soit entrepris pour elle.

[JT 29/08/09]

Dans cet échange coexistent deux motifs de justification de la réclamation de prescription de contention médicamenteuse. Celui qui émerge principalement concerne le travail des soignantes qui se voit contrarié par l'agitation de Mme Zermati et qu'on pourrait qualifier de "motif d'ordre » (Moreau, 2011, p.38), puisqu'il relève du bon fonctionnement du service. Le second, qui n'apparaît qu'à la fin de l'échange, correspond plutôt à un «motif thérapeutique » (Moreau, 2011, p.38) et porte sur les solutions à apporter directement à l'état de santé de la patiente en cours de dégradation.

La décision du médecin est surtout recherchée ici car elle vaut reconnaissance du besoin pratique des soignants d'avoir recours à la contrainte.

Après plusieurs mois, le médecin prend la décision de rendre systématique l'administration d'un traitement neuroleptique dans les périodes d' « agitation » de Mme Zermati.

Hélène Rollin, cadre de santé, résume le problème que pose la définition du moment à partir duquel le traitement médicamenteux doit être déclenché. Elle insiste sur l'importance pour les soignants de ne pas aller jusqu'à une situation de confrontation dans laquelle ils se trouveraient physiquement en danger. « Il fallait préciser quand on mettait en place ce protocole, dans quels cas. Les aidessoignants disaient : "quand elle est agitée". On travaille tous dans le même service mais on ne met pas toujours la même chose derrière un mot. Donc il faut préciser, 
leur faire comprendre que tout le monde ne comprend pas la même chose quand on écrit le mot "agité" [...] Mme Zermati par exemple, "agitée", c'est quand elle parle toute seule et se déplace dans le couloir ? Non, ça vous gérez. C'est quand elle vous insulte ? Ca aussi, vous gérez. C'est quand elle vous frappe, vous griffe ? C'est là que ça devient ingérable. C'est là qu'on a besoin des médicaments.» [ent. 16/08/09]

Dans cet extrait transparaît plus précisément encore l'intrication qui existe dans le recours à la prescription de contraintes entre leur fonction thérapeutique (les médicaments doivent apaiser Mme Zermati) et leur nécessité pratique du point de vue de l'organisation du travail (ils permettent aux soignants de réaliser les tâches prévues sans recevoir de coups). Les soignants reconnaissent aisément que les contentions mécaniques, comme les traitements médicamenteux, dont ils réclament la prescription, leur apparaissent dans certains cas comme indispensables moins pour des motifs médicaux qu'en raison des nécessité de la réalisation de leur travail. Les soins étant effectués " en séries », ils sont une grande partie de la journée dans les chambres et donc absents des espaces collectifs du service. Ils ne peuvent dès lors maintenir une surveillance constante sur l'ensemble des patients dont ils ont la responsabilité (entre 12 et 14 par aile de bâtiment). En cas de chute ou de «fugue» d'un patient, leur responsabilité professionnelle et celle des cadres de santé est engagée : leur hiérarchie ainsi que les membres de l'entourage familial peuvent leur demander des comptes. Pour éviter toute mise en cause, ils préfèrent donc voir les patients « agités » attachés à leur fauteuil par une ceinture pelvienne ou affaiblis par des médicaments, que de veiller, parallèlement aux actes de soins qu'ils effectuent dans les chambres, à ce qu'aucun incident n'ait lieu.

L'acte de prescription par le médecin, remplaçant les formes interstitielles de contrainte par des contentions officielles, vient structurer le corps à corps individuel entre soignants et patients. Il sert ainsi paradoxalement d'appui à un processus de désengagement du travail de contrainte et de sa responsabilité. Pour les soignants de première ligne, le désengagement est d'abord physique: les formes de contrainte prescrites, ayant pour effet de réduire largement les occasions de confrontation entre soignants et patients, supposent une implication corporelle beaucoup moins soutenue que les formes interstitielles. Il est aussi moral : quel que soit le jugement de chaque soignant sur la légitimité thérapeutique, pratique et morale, de la contrainte, il n'est pas 
question de le faire valoir dès lors que la responsabilité médicale et juridique de l’acte ordonné est portée par le médecin.

Ainsi, bien qu'elle soit parfois le résultat d'une sollicitation de certains soignants de première ligne, la prescription médicale de contention pacifie aussi les relations d'équipe, en rendant obligatoire le recours à la contrainte que peuvent sinon se reprocher les soignants entre eux (soit qu'ils le trouvent disproportionné par rapport à la situation, soit qu'ils désapprouvent un collègue qui n’assumerait pas sa part dans ce travail).

Karina Hernan est infirmière dans son pays d'origine. Son diplôme n'étant pas reconnu en France, elle exerce depuis plusieurs années comme aide-soignante dans différents établissements et a récemment été recrutée dans l'USLD, dont elle ne connaît pas encore tous les usages, étant encore stagiaire. Karina Hernan m'explique que lorsque Mme Rosier est arrivée dans le service, elle a eu l'impression que la contention avec une ceinture pelvienne était injustifiée. Elle avait lu dans son dossier que celle-ci n'était pas prescrite dans le service où Mme Rosier était hospitalisée auparavant et que, de surcroît, sa fille y était opposée. Le jour où Karina Hernan a dû s'occuper pour la première fois de Mme Rosier, elle ne l'a donc pas attachée avec la ceinture, pensant la surveiller durant toute la matinée, pour estimer s'il y avait besoin ou non de cette contention. Elle me raconte que la cadre de santé l'a reprise et lui a expliqué que le processus était inverse : elle ne pourrait retirer cette contention qu'une fois que le médecin aurait levé la prescription. En effet, l'ordonnance du médecin engage la responsabilité collective. Le service pouvant être tenu pour responsable de tout accident qui interviendrait alors qu'une mesure de contrainte était prescrite, personne ne doit se risquer à prendre l'initiative de ne pas appliquer l'ordonnance de contention. Karina Hernan m'exprime son étonnement : comment surveiller le fait que Mme Rosier ait vraiment besoin de cette contention (c'est à dire qu'il y a une risque de chute ou de «fugue») si on ne peut l'observer qu'attachée? Elle exécutera cependant la prescription et Mme Rosier restera sous contention durant quatre mois encore avant que le médecin ne lève la prescription [ent. 09/02/10]

La contrainte entre, grâce à l'acte de prescription, dans une organisation verticale où les lignes de décision sont stabilisées par la hiérarchie professionnelle qui prévaut ordinairement dans le travail hospitalier. 
Son accession au rang de pratique professionnelle légitime passe enfin par la manière dont elle est présentée à l'entourage familial des patients. A un premier niveau de discours, la prescription médicale de contention peut être affichée comme légitimité souveraine et servir de couverture pour garantir les soignants contre des accusations mettant en cause leur responsabilité personnelle (accusations qui peuvent aller d'un défaut de surveillance jusqu'à des actes de maltraitance, éventuellement soupçonnés face à des hématomes évoquant des coups). Un second niveau de discours laisse apparaître un autre enjeu : celui de l'établissement d'une hiérarchie entre, d'un côté, des exigences de sécurisation de la part de l'entourage jugées démesurées par les professionnels et, de l'autre, des motifs de recours à la contrainte estimés légitimes par l'équipe de soignants. La construction de cette légitimité passe en particulier par la démonstration de ce que cette pratique doit faire l'objet d'un cadre d'exercice strict, d'une évaluation et d'un suivi, que seuls les professionnels ont vocation à assurer.

L'une des cadres de santé est ainsi très attentive à ce que les contraintes prescrites soit proportionnées à la dégradation effective de l'état de santé de la patiente, affichant par là vis-à-vis de l'extérieur leur fonction thérapeutique qui s'impose à tout autre motif de décision. Elle insiste pour que l'équipe mette en place un suivi très transparent des différentes étapes et incidents qui l'amènent à opter pour un prescription de contention.

Alors que les relations avec les filles de Mme Zermati se tendent au fur et à mesure que se produisent des chutes ou des épisodes d'agressivité, Hélène Rollin, cadre de santé, réclame que tous les soignants soient très stricts sur le respect des prescriptions médicales, qui doivent s'imposer face aux demandes de la famille. «Vous n'êtes pas là pour exécuter les demandes de la famille, vous êtes là pour appliquer des prescriptions médicales. Dans le cas de Mme Zermati, vous ne devez pas répondre aux exigences de la famille qui met des petits mots sur les murs. En particulier en ce qui concerne les barrières du lit. Il n'y a pas de prescription médicale pour la barrière de gauche, donc vous ne mettez que la barrière de droite la nuit, même si la famille insiste. Parce que c'est une famille procédurière, qui n'hésitera pas à porter plainte s'il y a un accident ». Elle demande aussi aux aidessoignants d'écrire le plus d'informations possibles, avec leurs mots, dans le logiciel où figurent les transmissions d'une équipe à une autre, « pour se couvrir ». "Quand il y a une chute, vous donnez le plus d'éléments possible sur la situation : comment étaient les barrières [relevées ou non], est-ce qu'il y a du sang, est-ce que la 
patiente est face contre terre ou pas etc. » [JT 01/12/09]

L'usage qui est fait ici de la prescription médicale institue une hiérarchie entre savoirs professionnels et exigences profanes et inscrit alors formellement la contrainte dans l'ordre d'un travail assumé. Il normalise les relations entre l'équipe soignante et les familles de patients, en repoussant hors du cercle des interlocuteurs pertinents les nonprofessionnels, qui cherchent à faire valoir leur point de vue sur le niveau de contrainte acceptable dans le traitement de leur parent. Dans le cas de Mme Zermati, la cadre de santé estime que la recherche par la famille d'une sécurisation maximale par la mise en place de deux barrières au lit est plus dommageable à la patiente (qui, en tentant de les enjamber, fait des chutes impressionnantes) que le risque qu'elle court avec un niveau de contention moindre (une seule barrière).

L'inscription de la contrainte dans un cadre professionnel dominé par la légitimité médicale, par le biais de sa traduction en prescriptions formelles, n'épuise cependant pas les débats qui traversent l'équipe et certains soignants manifestent des ambivalences vis-à-vis du sens qu'ils donnent à cette activité.

2.3 «Et si elle tombe, c'est la vie!»: critiques et ambivalences vis-à-vis de la contrainte médicalement ordonnée

Si pour des raisons pratiques d'organisation, la prescription de contentions mécaniques ou de médicaments psychotropes facilite et allège le travail des soignants de première ligne, une partie d'entre eux se montre néanmoins critique vis-à-vis de ce mode de gestion des «troubles du comportement», soulignant notamment le fait que le traitement hospitalier lui-même pourrait être à l'origine de ces troubles.

Manuelle Sallet, aide soignante, m'explique: "Y'a certains médicaments que les patients seraient pas obligés de prendre si on avait du personnel. Ils seraient pas agités si on avait du temps avec eux, pour leur parler. On remplace le personnel par des médicaments » [ent. 29/08/09]

Au delà de ces regrets diffus, des critiques situées émergent dans le cours de l'action, provenant principalement d'infirmiers, qui se trouvent dans une position intermédiaire, entre aides-soignants et médecin. S'ils sont impliqués de manière moins intensive que les aides-soignants dans la surveillance et la gestion des comportements inadaptés à 
l'organisation du travail hospitalier, ils représentent tout de même des référents pour ces derniers dans les situations quotidiennes où le besoin de contrainte se fait sentir. A ce titre, ils sont à la fois les garants de l'application stricte des prescriptions médicales et responsables de trouver des solutions pratiques qui permettent aux aides-soignants de poursuivre leur activité.

Un après-midi, Mme Pougny est particulièrement agitée et déambule dans les couloirs de manière inhabituelle. Françoise Sylvestre, l'aide-soignante qui est en charge de la salle, la perd de vue plusieurs fois et s'inquiète. Lorsqu'elle la retrouve finalement, elle annonce, excédée de devoir courir après elle : «Bon, je vais la mettre au lit parce que sinon elle va fuir ! ». Inès Dahmani, l'infirmière, la reprend fermement: « Non non, Mme Pougny elle a pas de barrières à son lit, elle a que la gauche. Tu sais ce que tu fais ? Tu l'installes dans son fauteuil et puis tu lui mets sa tablette devant, tu lui dis qu'elle va avoir son goûter ». L'infirmière se justifie auprès de moi : «Je veux pas qu'on me cale les patients au lit ! ». [JT 10/08/09]

Cette infirmière exprime ici une préférence pour des formes interstitielles de contrainte qui lui paraissent moins dommageables pour une patiente qu'elle craint de voir confinée au lit par une extension (non prescrite d'ailleurs) de l'usage des contentions médicales. Par ailleurs, mettant en œuvre les ordonnances de contention par l'administration des médicaments, les infirmiers disposent d'une certaine marge d'autonomie dans l'application effective de la prescription.

Hatem Rabo, l'un des médecins du service, est réputé, parmi les soignants de l'équipe, faire un usage systématique des médicaments psychotropes pour apporter une réponse à un large éventail de comportements. Dans la période où les tentatives de sortie de M. Nanteuil s'accentuent, il m’explique «Là je lui ai donné Equanil Equanil Equanil, Equanil [anxiolytique] jusqu’à épuisement! Et puis si ça suffit pas je le mettrai sous Risperdal [neuroleptique] ! »

Quand Ines Dahmani, l'infirmière qui prend la relève, est prévenue que le médecin a prescrit une dose supplémentaire d'Equanil à M. Nanteuil, elle refuse de la lui administrer, estimant qu'il en a déjà reçu suffisamment : "Il en a eu 400mg à $10 \mathrm{~h} 30$ et $400 \mathrm{mg}$ à $12 \mathrm{~h}$ ? Moi je lui en donne pas à $18 \mathrm{~h}$ s'il en a eu déjà $800 \mathrm{mg}$ dans la journée ! » [JT 10/08/09]

Là encore, la prescription médicale lui paraissant démesurée, l'infirmière module la contrainte médicamenteuse dans les limites de ce qu'il lui semble acceptable de réaliser 
elle-même.

S'ils sont critiques, les infirmiers ne discutent pourtant pas directement les prescriptions médicales. D'autres intervenants se mettent en revanche plus ouvertement en position de négocier les choix de contention. La représentante de l'accueil de jour intervient en réunion sur le cas des patients que son équipe reçoit deux fois par semaine pour des activités thérapeutiques. Elle met l'accent sur la gravité des conséquences que peuvent avoir les mesures de contrainte sur l'état de santé des personnes concernées. Elle se place régulièrement du côté de l'allègement de ces dernières, soulignant l'efficacité des moyens humains pour remplacer la contrainte, en particulier la contention médicalement prescrite (rappelons que ces moyens humains sont beaucoup plus importants à l'accueil de jour que dans le reste des services d'hébergement de l'hôpital). Ces conséquences ne sont pas méconnues dans l'USLD et sont envisagées dans les réunions d'équipe. Même dans les cas où ils agissent positivement sur l'agitation, les traitements médicamenteux et les contentions mécaniques sont connus pour faire courir le risque d'accélérer la dégradation de l'état physique. Les neuroleptiques sont réputés par les instances de régulation de la pratique médicale (ANAES, 2000) avoir un effet déstabilisateur sur l'équilibre des patients, qui, sous traitement, ont tendance à faire des chutes plus nombreuses. De même, la ceinture pelvienne, en maintenant la personne assise toute la journée dans son fauteuil, l'empêche de se mouvoir et est responsable d'une perte de masse musculaire à la fois nécessaire à la marche et entretenue par cette dernière

Pour interrompre le cycle des chutes de Mme Zermati, le médecin lui prescrit successivement un traitement neuroleptique, puis, pour compenser le danger de perte d'équilibre, une ceinture pelvienne. Lors des réunions d'équipe, au cours desquelles sont prises ces décisions successives, est évoquée la gravité du choix qui doit être fait :

Hélène Rollin, cadre de santé : elle se dégrade très très vite cette dame, depuis juillet, c'est un puits sans fond...

Viviane Bartolo, infirmière : dès qu'elle se lève, il faut être avec elle.

Hatem Rabo, médecin : comme on lui a mis une contention au fauteuil, elle va s'atrophier. Mais il faut faire un choix ! [JT 24/11/09]

Un mois et demi plus tard, le médecin, constatant que la dégradation physique qu'il 
anticipait a bien eu lieu, décide de maintenir la contention.

Hélène Rollin, cadre de santé : je crois qu'il faut maintenir la contention au fauteuil. Maintenant qu'elle ne marche plus, elle risque une faute musculaire. Mais les chutes étaient trop violentes, on ne peut pas se permettre d'en risquer à nouveau. Nathalie Laborde, éducatrice spécialisée à l'accueil de jour: mais quand on l'accompagne, elle marche...

Hatem Rabo, médecin : on maintient la contention. [JT 05/01/10]

Deux mois plus tard, l'état de Mme Zermati est réévalué en réunion.

Nathalie Laborde, éducatrice spécialisée à l'accueil de jour: on essaye de la mobiliser pour qu'elle fasse quelques pas, mais maintenant elle ne marche plus sur les talons. On a l'impression qu'à force d'être au fauteuil, elle s'est rétractée. Nous on se demandait si on pourrait pas faire une demande de kiné pour elle?

Hatem Rabo, médecin : c'est contradictoire de demander un kiné alors qu'on lui met une ceinture pelvienne.

Hélène Rollin, cadre de santé : la marche est de plus en plus dangereuse pour elle, donc on l'accompagne dans les quelques mouvements qu'elle peut faire, mais sans plus. [JT 09/03/10]

Cherchant à éviter le risque de chute, le médecin et l'équipe prennent donc des décisions, se traduisant par des prescriptions successives, qui accélèrent la grabatérisation, c'est à dire le processus qui amène progressivement le patient à ne plus pouvoir se mouvoir ou se lever spontanément.

Une infirmière souligne ce paradoxe des pratiques de l'équipe qu'elle rend responsable, par un recours abusif à des prescriptions de contention, de l'augmentation du risque de dégradation d'une patiente pourtant en bonne condition physique.

Mme Rosier fait l'objet d'une prescription de contention dans son fauteuil depuis qu'elle est entrée dans le service. Après plusieurs semaines de prise en charge hebdomadaire par la psychomotricienne, il s'avère que Mme Rosier marche bien, sans besoin d'aide, et qu'elle est désireuse de pouvoir se déplacer seule. Une levée de cette contention est donc envisagée. Les soignants de première ligne sont très réticents à cette idée et ils évoquent le risque de chute pour justifier leur réaction. [JT 24/11/09] 
Elisa Tomas, infirmière, résume ainsi sa position personnelle sur ce cas : « Je pense que dans le cas de Mme Rosier, il n'y a pas d'indication [pour prescrire une ceinture pelvienne] puisqu'elle n'est pas grabataire. Il faudrait la laisser déambuler librement. Et si elle tombe, c'est la vie ! Elle devient grabataire mais c'est la vie. [...] Alors que si on l'attache et qu'elle devient grabataire parce qu'elle peut plus se lever, c'est pas normal ». [JT 10/11/09]

Pour justifier les choix en matière de contrainte, la légitimité médicale des prescriptions de contention apparaît donc insatisfaisante. Vis-à-vis de l'extérieur, elle est brandie comme souveraine et, s'imposant à toute velléité d'intervention de l'entourage, elle permet de «se couvrir». En interne, le recours à la contention prescrite est justifié par l'inadaptation de l'organisation hospitalière du travail aux comportements de certains patients. Pourtant la prescription ne semble pas tarir toutes les questions qui émergent dans le cours quotidien de la prise en charge. Celle de la mise en œuvre d'une contrainte juste et mesurée au regard d'autres normes professionnelles que celle de la protection contre les risques (de dégradation des patients, de judiciarisation etc.) reste ouverte. La négociation dans l'équipe, organisée autour de l'alternative entre prescription de contention et absence de prescription, laisse en effet dans l'ombre l'éventail des formes de contrainte plus discrètes, qui sont pourtant mobilisées au quotidien sans être discutées de manière approfondie.

\section{Conclusion}

Les pages qui précèdent ont cherché à alimenter la réflexion sur la contrainte en tant qu'elle participe aujourd'hui de la prise en charge des personnes âgées. En la considérant non pas dans les termes habituels qui opposent des pratiques thérapeutiques officielles (la contention) à des déviances condamnables (la maltraitance), il s'agissait ici de saisir la contrainte sous l'angle du travail qu'elle représente dans l'activité quotidienne de soignants hospitaliers en USLD et de décrire un certain nombre des caractéristiques de ce travail. On a pu montrer que le travail de contrainte représente une part non négligeable de l'activité des professionnels de l'USLD, ce qui permet d'admettre qu'il relève de l'ordinaire du traitement gériatrique. Seule une partie des tâches qui lui sont associées est officiellement réglementée 
(formes prescrites), quand d'autres ne sont pas expressément décrites (formes interstitielles). Dans un contexte de reconnaissance partielle de ce pan de l'activité gériatrique hospitalière, une division informelle du travail affecte prioritairement au bas de l'échelle la part non prescrite des tâches de contrainte, entérinant son caractère de «sale boulot». Cette division organisationnelle du travail est redoublée d'une hiérarchisation morale des objectifs servis par les différents acteurs professionnels. D’un côté, les soignants de première ligne, les plus impliqués dans la gestion quotidienne des patients et en particulier des débordements que représentent les comportements perturbants, font valoir de manière plus ou moins explicite la nécessité face à laquelle ils se trouvent d'obtenir la disponibilité des corps des patients pour les soins, dans les temporalités qui permettent le fonctionnement fluide du service. De l'autre, les soignants de seconde ligne défendent la primauté de l'intention thérapeutique, condamnant par une hiérarchisation implicite des objectifs toute forme de traitement qui rendrait prioritaires les nécessités propres à l'activité institutionnelle.

Plus qu'à rendre visible le fonctionnement de l' «institution totale» à l'œuvre (Goffman, 2005, [1968]), l'article a visé surtout à restituer l'existence d'une hétérogénéité dans les manières dont une équipe de professionnels pratique et s'approprie la contrainte comme modalité de l'activité hospitalière. On a ainsi pu saisir la place des interactions quotidiennes, à travers la mise en lumière de négociations, qui contribuent à légitimer dans le quotidien le travail de contrainte sous certaines de ses formes. En effet, on a vu que, si les discussions sont organisées par la question du recours ou non à la prescription médicale de contention, cette dernière n'épuise pas les sources de contestation et qu'elle ne règle pas pour les professionnels les ambivalences qu'ils entretiennent vis-à-vis du travail de contrainte.

Le monde gériatrique n'a pas, comme la psychiatrie par exemple, de tradition propre de réflexion et de controverse sur le rôle de la contrainte dans le traitement, qui peut, sous certaines conditions, excéder une simple logique de gestion des comportements perturbants et s'inscrire dans une perspective thérapeutique. Ce secteur professionnel vit cependant depuis une dizaine d'années des évolutions importantes. On peut penser que la montée en charge de la question des droits et du consentement des patients/résidents d'une part et l'émergence d'un domaine spécialisé dans la prise en charge de la maladie d'Alzheimer d'autre part (caractérisé notamment par la création 
de structures fermées) contribuent à faire sortir les négociations dont il a été question ici de leur invisibilité et à inscrire plus formellement la question des usages de la contrainte en gériatrie dans l'espace des débats professionnels.

\section{Bibliographie :}

\section{Ouvrages et articles}

Arborio, AM., 2001. Un personnel invisible : les aides-soignantes à l'hôpital. Anthropos, Paris.

Avril, C., Cartier, M. et Serre, D., 2010. Enquêter sur le travail : concepts, méthodes, récits. La Découverte, Paris.

DRESS, février 2005. Les établissements d'hébergement pour personnes âgées en 2003 : activité et personnel premiers résultats de l'enquête EHPA 2003. Etudes et résultats 379.

DRESS, août 2009. L'offre en établissements d'hébergement pour personnes âgées en 2007. Etudes et résultats 689.

Drulhe, M., 2000. Le travail émotionnel dans la relation soignante professionnelle, un point de vue au carrefour du travail infirmier. In: Cresson, G., Schweyer, FX., (Eds) Professions et institutions de santé face à l'organisation du travail : aspects sociologiques. Editions de l'ENSP, Rennes, pp. 15-29.

Dujarier, MA., 2002. Comprendre l'inacceptable : le cas de la maltraitance en gériatrie. Revue internationale de Psychosociologie VIII (19), 111-124.

Dujarier, MA. et Gaulejac, V., 2006. L'idéal au travail. Presses universitaires de France, Paris.

Fassin, D., 2009. Les économies morales revisitées. Annales. Histoire, Sciences Sociales 64 (6), 1237-1266.

Foucault, M., 1993 [1975]. Surveiller et punir : naissance de la prison. Gallimard, Paris.

Gérontologie et Société, 2010. Pour une bientraitance : faut-il repenser le soin ?. Gérontologie et société 2 (133).

Goffman, E., 2005 [1968]. Asiles : études sur la condition sociale des malades mentaux. Les Editions de Minuit, Paris. 
Hacking, I., 1988. The Sociology of Knowledge About Child Abuse. Noûs 22 (1), 53-63.

Hochschild, AR., 1979. Emotion Work, Feeling Rules, and Social Structure. American Journal of Sociology 85 (3), 551-575.

Hughes, E., 1996. Le regard sociologique : essais choisis. Éditions de l’EHESS, Paris.

Laforgue, D., 2009. Pour une sociologie des institutions publiques contemporaines. Socio-logos 4.

Leibing, A., 2009. Tense Prescriptions? Alzheimer Medications and the Anthropology of Uncertainty. Transcultural Psychiatry 46 (1), 180-206.

Mallon, I., 2004. Vivre en maison de retraite : le dernier chez-soi. Presses universitaires de Rennes, Rennes.

Mauss, M., 1968 [1934], Sociologie et anthropologie. Presses Universitaires de France, Paris.

Mercadier, C., 2002. Le travail émotionnel des soignants à l’hôpital : le corps au cœur de l'interaction soignant-soigné. Seli Arslan, Paris.

Molinier, P., 2006. Le care à l'épreuve du travail. Vulnérabilités croisées et savoir-faire discrets. In : Paperman, P. et Laugier, S., Le souci des autres. Éthique et politique du care. Editions de l'EHESS, Paris, pp. 299-316.

Molinier, P., 2009. Vulnérabilité et dépendance : de la maltraitance en régime hospitalier. In : Jouan, M. et Laugier, S. (Eds), Comment penser l'autonomie ? Entre compétences et dépendances. Presses Universitaires de France, Paris, pp. 433-458.

Moreau, D., 2010. Contraindre pour soigner? Le care à l'épreuve de la contrainte dans un service d'hospitalisation psychiatrique. In Crignon-De Oliveira, C. et Gaille, M., Qu'estce qu'un bon patient? Qu'est-ce qu'un bon médecin ? Réflexions critiques, analyses en contexte et perspectives historiques. Seli Arslan, Paris, pp. 168-181.

Moreau, D., 2011. Conjurer la qualification de violence dans l'usage professionnel de la contrainte : le recours à la chambre d'isolement dans un service de psychiatrie. Revue Sociologie Santé (33), pp.29-47.

Paperman, P. et Laugier, S., 2006. Le souci des autres. Éthique et politique du care. Editions de l'EHESS, Paris.

Rimbert, G., 2005. Le chronomètre et le carillon. Temps rationalisé et temps domestique en maison de retraite. Lien social et Politiques 54, 93-104. 
Rimbert, G., 2008. Concilier soin et réparation : le soutien humaniste aux personnes âgées dépendantes. Sociologie du Travail, 50 (4), 521-536.

Rimbert, G., 2010. Vieillards sous bonne garde: réparer l'irréparable en maison de retraite. Editions du Croquant, Broissieux.

Strauss, A., Fagerhaugh, S., Suczek, B. et Wiener, C., 1982. Sentimental work in the technologized hospital. Sociology of Health and Illness 4 (3), 254-278.

Vigarello, G., 2005. L'intolérable de la maltraitance infantile. Genèse de la loi sur la protection des enfants maltraites et moralement abandonnés en France. In : Bourdelais, P. et Fassin, D. (Eds). Les Construction de l'intolérable: études d'anthropologie et d'histoire sur les frontières de l'espace moral. La Découverte, Paris.

\section{Rapports et littérature professionnelle :}

ANAES, 2000. Evaluation des pratiques professionnelles dans les établissements de santé. Limiter les risques de la contention physique de la personne âgée.

ANESM, 2008. La bientraitance : définitions et repères pour la mise en œuvre. Recommandations de bonnes pratiques professionnelles.

Brown, H., 2002. La protection des adultes et enfants handicapés contre les abus. Rapport pour le Conseil de l’Europe, La Documentation Française, Paris.

Compagnon, C. et Ghadi, V., 2009. La maltraitance "ordinaire" dans les établissements de santé : Etude sur la base de témoignages. Rapport pour la Haute Autorité de Santé, Paris.

Deliot, C., Caria, A. et Boiteux, C., 2009. Éthique: enjeux de la diffusion des notions de bientraitance et de maltraitance. Gestions hospitalières 491, 593-597.

Gineste, Y. et Pellissier, J., 2007. Humanitude : comprendre la vieillesse, prendre soin des Hommes vieux. Armand Colin, Paris.

HAS et FORAP, 2012. Le déploiement de la bientraitance : guide à destination des professionnels en établissements de santé et EHPAD.

Hugonot, R., 2007. Violences invisibles : reconnaître les situations de maltraitance envers les personnes âgées. Dunod, Paris.

Juilhard, JM., Blanc, P., 2003. Maltraitance envers les personnes handicapées : briser la loi du silence. Rapport de la commission d'enquête n³39 du Sénat Français. 


\section{Chartes}

Direction Générale de la Santé, Direction de l'hospitalisation et de l'organisation des soins, 2006. Charte des droits du patient hospitalisé.

Fondation Nationale de Gérontologie, 2007, Charte des droits et libertés de la personne âgée en situation de handicap et de dépendance. 